\title{
The glyoxylate cycle and alternative carbon metabolism as metabolic adaptation strategies of Candida glabrata: perspectives from Candida albicans and Saccharomyces cerevisiae
}

\author{
Shu Yih Chew, Wallace Jeng Yang Chee and Leslie Thian Lung Than *i)
}

\begin{abstract}
Background: Carbon utilization and metabolism are fundamental to every living organism for cellular growth. For intracellular human fungal pathogens such as Candida glabrata, an effective metabolic adaptation strategy is often required for survival and pathogenesis. As one of the host defence strategies to combat invading pathogens, phagocytes such as macrophages constantly impose restrictions on pathogens' access to their preferred carbon source, glucose. Surprisingly, it has been reported that engulfed C. glabrata are able to survive in this harsh microenvironment, further suggesting alternative carbon metabolism as a potential strategy for this opportunistic fungal pathogen to persist in the host.

Main text: In this review, we discuss alternative carbon metabolism as a metabolic adaptation strategy for the pathogenesis of C. glabrata. As the glyoxylate cycle is an important pathway in the utilization of alternative carbon sources, we also highlight the key metabolic enzymes in the glyoxylate cycle and its necessity for the pathogenesis of $C$. glabrata. Finally, we explore the transcriptional regulatory network of the glyoxylate cycle.

Conclusion: Considering evidence from Candida albicans and Saccharomyces cerevisiae, this review summarizes the current knowledge of the glyoxylate cycle as an alternative carbon metabolic pathway of C. glabrata.
\end{abstract}

Keywords: Candida, Candida glabrata, Carbon metabolism, Glyoxylate cycle, Metabolic adaptation, Pathogenesis, virulence, yeast.

\section{Introduction}

Fungi are a group of eukaryotic organisms that can provoke a broad range of infectious diseases in humans, ranging from mild superficial infections to potentially life-threatening systemic infections. Candida species, such as Candida albicans and Candida glabrata, are ubiquitous and are considered benign residential microflora in the human body. However, an increased presence of immunocompromised individuals in human populations can contribute to the spread of candidiasis

\footnotetext{
* Correspondence: leslie@upm.edu.my

Department of Medical Microbiology and Parasitology, Faculty of Medicine and Health Sciences, Universiti Putra Malaysia, 43400 UPM Serdang, Selangor, Malaysia
}

(c) The Author(s). 2019 Open Access This article is distributed under the terms of the Creative Commons Attribution 4.0 International License (http://creativecommons.org/licenses/by/4.0/), which permits unrestricted use, distribution, and reproduction in any medium, provided you give appropriate credit to the original author(s) and the source, provide a link to the Creative Commons license, and indicate if changes were made. The Creative Commons Public Domain Dedication waiver (http://creativecommons.org/publicdomain/zero/1.0/) applies to the data made available in this article, unless otherwise stated.

infections [1-3]. Invasive candidiasis is common among intensive care unit (ICU) and organ transplant patients and is often associated with increased costs of care, significant reductions in lifespan and prolonged hospitalization even after receiving antifungal treatments [4-8].

Bloodstream infections (BSIs) caused by Candida species or candidaemia are the most common manifestation of invasive candidiasis. In recent years, a shift of predominance from $C$. albicans to non-C. albicans Candida (NCAC) species has been observed, and C. albicans now accounts for only half of the invasive candidiasis cases reported [9]. Notably, C. glabrata has been recognized as either the second or third most common cause of invasive candidiasis after C. albicans [10], and similar 
epidemiological data have also been highlighted in global surveillance programmes such as SENTRY, ARTEMIS and TRANSNET [11-14]. Pfaller et al. (2014) reported that invasive candidiasis caused by C. glabrata is most commonly associated with patients who receive treatment for solid tumours or undergo solid organ transplantation (SOT) [15]. Among the patients with solid tumours and infections caused by NCAC species, $53.8 \%$ were caused by C. glabrata. In addition, C. glabrata was the main aetiological agent of $63.7 \%$ of SOT patients with NCAC infections. Overall, the increasing prevalence of NCAC species, particularly C. glabrata, is alarming due to the high mortality rates associated with such infections; thus, there is an urgent need to improve the current diagnostic and therapeutic options for better management of fungal BSIs [16].

Host immunity was believed to be the main factor responsible for the establishment of opportunistic fungal infections caused by yeast pathogens. However, this concept has changed, and it is now agreed that the pathogenicity of these yeast pathogens also plays a crucial role in infection [17]. For instance, virulence factors and fitness attributes such as metabolic flexibility, adherence to the host epithelia, biofilm formation, phenotypic switching, filamentation, secretion of hydrolytic enzymes, production of haemolysin and production of extracellular hydrolase are all associated with Candida species $[18,19]$. Relative to $C$. albicans, the virulence factors and fitness attributes of $C$. glabrata are not well known, particularly in its natural niche.

The invasion strategy of C. glabrata is believed to be independent of phenotypic switching, as opposed to C. albicans, which employs invasion mechanisms that involve active penetration through hyphae extension and induced endocytosis [20,21]. Despite being a relatively less virulent Candida species and generally having a weaker tissuedamaging ability compared to C. albicans, C. glabrata is still able to breach the natural barriers, invade the human bloodstream and cause systemic infection, presumably through SOT, parenteral nutrition, trauma, surgical or indwelling medical devices $[8,22]$. In addition, C. glabrata uses a co-infection approach in infecting the human bloodstream, often by exploiting the tissue invasion and damage of the natural barriers caused by other Candida species, such as C. albicans [21, 23, 24].

Upon entering the human bloodstream, C. glabrata triggers a weaker polymorphonuclear neutrophil activation that primarily induces the recruitment of monocytic cells to the site of infection and triggers monocyte engulfment [25]. Since C. glabrata can survive and propagate within macrophages but not in neutrophils [26, 27], this fungal pathogen could use monocytes or macrophages as "Trojan horses" to gain protection against host defence mechanisms, particularly against neutrophil attack $[25,26]$. In fact, C. glabrata clearly pursues different immune evasion and persistence strategies compared to $C$. albicans, as escape from the macrophages upon engulfment is not the priority of this fungal pathogen. Unlike C. albicans, C. glabrata can persist and replicate within macrophages without causing any significant damage to the host until the host cells eventually burst and release the fungal cells [26].

While trapped within macrophages, C. glabrata must rely on endogenous resources for survival because macrophages are often depicted as glucose-deficient [28, 29]. It has been shown that the ability of C. glabrata to mobilize intracellular resources through autophagy serves as a major contributor to sustaining the viability of this pathogen during carbon starvation [30,31]. In addition to recycling intracellular resources via autophagy, the ability to utilize carbon sources other than glucose could potentially assist in the survival of engulfed C. glabrata. This review aims to discuss the metabolic adaptation and alternative carbon metabolism in C. glabrata. Taking cues from Saccharomyces cerevisiae and C. albicans, this review also discusses the role of the glyoxylate cycle in alternative carbon metabolism as well as the transcriptional regulation of this anabolic pathway in C. glabrata.

\section{Metabolic adaptation strategy in C. glabrata}

Nutrients are crucial for all living organisms, including fungi. Since Candida species often inhabit host niches with different nutrient availability, these fungal pathogens must be equipped with a high degree of metabolic flexibility and possess metabolic adaptation mechanisms that are required for effective nutrient acquisition [32]. Despite being a common human pathogen and rarely being found in environmental niches, Candida species such as C. albicans and C. glabrata still retain an impressive degree of metabolic flexibility [33, 34]. In addition, the virulence factors and fitness attributes of these fungal pathogens are firmly dependent on fungal metabolism, strengthening the truism of "you are what you eat" [35].

Transcriptional analyses of macrophage-engulfed $C$. albicans and C. glabrata revealed extensive metabolic reprogramming that reflects nutrient deprivation $[28,29]$. These effects include the upregulation of genes that encode key metabolic enzymes in three interconnected anabolic pathways for alternative carbon metabolism, i.e., gluconeogenesis (PCK1 and FBP1), glyoxylate cycle (ICL1 and MLS1) and $\beta$-oxidation of fatty acids (FOX2, POT1 and $P O X 1)$, coupled with the downregulation of genes related to glycolysis and translational machinery (e.g., elongation factors, ribosomal protein genes, translation initiation and tRNA synthetases). The induction of these key enzyme genes suggests that the microenvironment within macrophages is deficient in glucose; however, the 
confined C. glabrata cells are still able to utilize endogenous resources such as alternative carbon sources for their growth and survival. Furthermore, Rai et al. (2012) reported that engulfed C. glabrata cells primarily utilize acetyl coenzyme A (acetyl-CoA) from the breakdown of fatty acids via the glyoxylate cycle as the main carbon source for the production of cellular building blocks [36].

Other than macronutrients, fungal pathogens are also dependent on micronutrients for the full functionality of proteins and enzymes, which are essential for survival and host colonization [32]. Trace metals such as iron are usually sequestered from fungal pathogens via nutritional immunity, forcing these fungal pathogens to employ iron acquisition mechanisms during iron starvation. In Candida species, C. albicans is able to obtain xenosiderophores from other microorganisms through iron parasitism using the Sit1 siderophore transporter [37]. Iron parasitism and the Sit1 transporter are well conserved in many fungal species, such as $S$. cerevisiae, Cryptococcus and Aspergillus [38]. Nevertheless, C. glabrata is unable to use siderophores of bacterial origin for iron uptake. In fact, C. glabrata can only exploit fungal xenosiderophores via the Sit1 homologue transporter, which is critical for its survival in macrophages [39]. To date, the uptake of other important trace metals in C. glabrata, such as copper and manganese, remains uncharacterized.

In addition to reprogramming its metabolic activity, $C$. glabrata obtains nutrients through mobilization of intracellular resources via a specialized autophagy known as pexophagy. Deletion of the genes required for pexophagy, ATG11 and ATG17, rendered C. glabrata more susceptible to macrophage killing. Therefore, pexophagy could potentially assist trapped C. glabrata in overcoming transient periods of nutrient deprivation in the phagosome and facilitate disseminated infection [30].

\section{Alternative carbon metabolism in C. glabrata}

Glucose remains the preferred carbon source for Candida species, and it is an important carbon and energy source for cell growth and development. Generally, glucose sensing and uptake in Candida species involve three main pathways, including the sugar receptorrepressor pathway, adenylate cyclase pathway and glucose repression pathway [40]. However, the availability of glucose varies in many host niches and anatomical sites, ranging from $0.05-0.1 \%$ in vaginal secretion to $0.1-0.2 \%$ in the blood $[41,42]$. Apart from glucose, yeast cells are able to utilize alternative carbon sources, including but not limited to lactate, acetate, ethanol, glycerol and oleate [43]. Organic and short-chain fatty acids (SCFAs), such as acetate and lactate, are produced by intestinal bacteria from the degradation of complex carbohydrates [42-44]. Acetate and lactate can also be found in other host niches, such as the vaginal environment, which is dominated by vaginal microflora. Previously, it has been shown that $C$. glabrata can survive in carbon starvation conditions upon macrophage engulfment [30]; therefore, it is likely that alternative carbon metabolism plays a major role in the survival of C. glabrata trapped within macrophages.

Glycerol is utilized by yeast cells as an important carbon source as well as for osmoregulation [45]. In fact, glycerol is also one of the alternative carbon sources that can be utilized by C. glabrata, and the uptake process is probably mediated by the glycerol transporter encoded by FPS1 and FPS2 [46]. Glycerol is first converted to glycerol-3-phosphate by the glycerol kinase Gut1, followed by conversion to dihydroacetone phosphate (DHAP), catalysed by glycerol-3-phosphate dehydrogenase (Fig. 1). Subsequently, DHAP can enter gluconeogenesis through conversion into glyceraldehyde 3phosphate by triose phosphate isomerase. On the other hand, lactate is transported through a monocarboxylate permease encoded by JEN1 in yeast such as $S$. cerevisiae and $C$. albicans. The presence of glucose represses the expression of JEN1, and the degradation of proteincoding mRNA is also observed when induced JEN1 is exposed to glucose [47, 48]. Lactate is converted to pyruvate by two different oxidoreductases encoded by CYB2 and DLD1 [49], which serves as an intermediate for gluconeogenesis. Although JEN1 is absent in C. glabrata [50], lactate dehydrogenase-mediated lactate assimilation has been shown to be vital for the survival and adaptation of C. glabrata in the mouse intestine [51].

Ethanol is believed to enter the cells through passive diffusion, whereas acetate is transported to the cells through the carboxylate transporter acetate permease [54-56]. Both carbon sources are ultimately converted to acetyl-CoA by acetyl-CoA synthetase (Acs1) in the cytosol (Fig. 1). It has been shown that C. glabrata are able to utilize acetate, even in the presence of glucose $[57,58]$. In addition, the putative acetate permease gene $A D Y 2$ was induced in C. glabrata in response to engulfment by macrophages [29] and neutrophils [59]. These observations suggest that acetate is relevant in vivo and that C. glabrata may assimilate acetate or other SCFAs in microenvironments with poor glucose availability. In addition, transporters such as Fat1, Faa1 and Faa4 are believed to be involved in the uptake of fatty acids in yeast cells [60]. Orthologous genes that encode these fatty acid transporters have been identified in C. glabrata, although they have not been fully characterized. Unlike ethanol and acetate, fatty acids are broken down to acetyl-CoA via $\beta$-oxidation in the peroxisomes, which involves the enzymes fatty acyl-CoA oxidase, 3hydroxyacyl-CoA dehydrogenase and 3-oxoacyl-CoA thiolase. Acetyl-CoA generated from the breakdown of acetate, ethanol and fatty acids fuels the glyoxylate cycle 


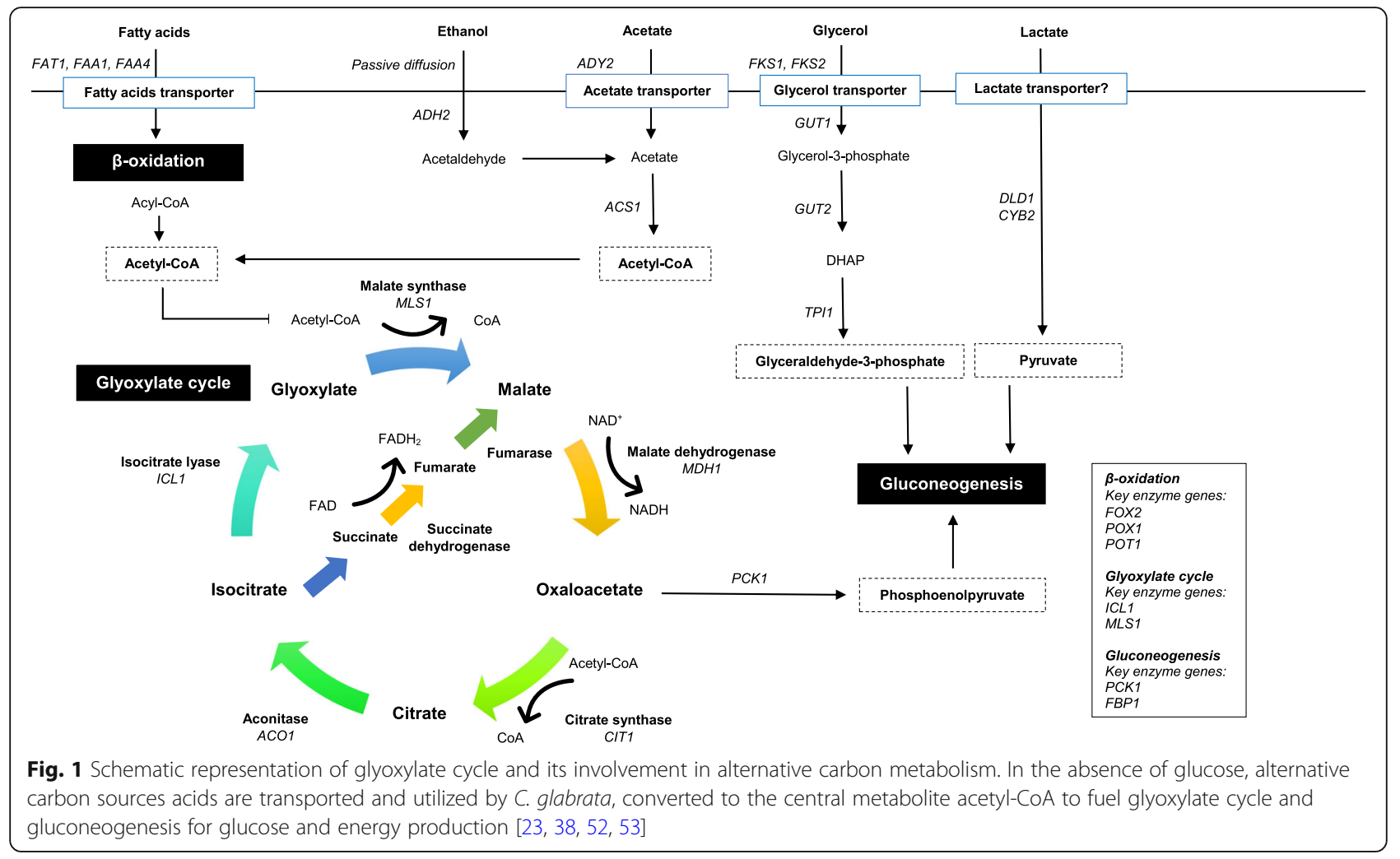

and gluconeogenesis for glucose production when glucose availability is scarce (Fig. 1) [28, 43].

The breakdown of alternative carbon sources, such as fatty acids, ethanol and acetate, leads to the generation of the central metabolite acetyl-CoA in different subcellular compartments [52]. Specifically, breakdown of fatty acids generates acetyl-CoA that is solely peroxisomal, while breakdown of ethanol and acetate produces cytosolic acetyl-CoA. Due to its bulkiness and amphiphilic nature, transport of peroxisomal and cytosolic acetyl$\mathrm{CoA}$ to the glyoxylate cycle often requires a functional acetyl unit transport system [52, 61]. In general, S. cerevisiae appears to use two parallel modes of acetyl unit transportation systems, namely, the carnitine shuttle and citrate synthase pathway, while $C$. albicans exclusively depends on the carnitine shuttle [reviewed in [52]]. The genome of C. glabrata also encodes three still uncharacterized, putative carnitine acetyltransferase orthologues, Cat2, Yat1 and Yat2, which are similar to those in S. cerevisiae and C. albicans $[62,63]$, suggesting that a functional carnitine shuttle also exists in C. glabrata for acetyl-CoA transportation. It has been shown that $C$. albicans is fully dependent on the carnitine shuttle for transportation of acetyl-CoA due to the absence of peroxisomal citrate synthase (Cit2), and this is further underlined by the fact that $C$. albicans also possesses a complete carnitine biosynthesis pathway [64-66]. In contrast to C. albicans, a putative Cit2 orthologue exists in C. glabrata (60\% similarity to S. cerevisiae Cit2), suggesting that $C$. glabrata may utilize two modes of acetyl unit transportation, similar to the phylogenetically more closely related $S$. cerevisiae. Nonetheless, further investigation is needed to fully decipher the carnitine shuttle and potential peroxisomal citrate synthase pathway in C. glabrata.

\section{Glyoxylate cycle: an overview}

The glyoxylate cycle is an anaplerotic variant of the tricarboxylic acid (TCA) cycle and is an anabolic pathway occurring in most protists, plants, bacteria and fungi. The glyoxylate cycle is assumed to be absent in animals and human tissues, with nematodes at the early stages of embryogenesis being the only known exception [67, 68]. The primary function of the glyoxylate cycle is to allow growth when glucose is not available and two-carbon compounds, such as ethanol and acetate, are the only sources of carbon [69]. The glyoxylate cycle is basically a shunt in the TCA cycle, with which it shares many metabolic enzymes, including malate dehydrogenase, citrate synthase, and aconitase [70]. The two decarboxylation steps involve isocitrate dehydrogenase and $\alpha$-ketoglutarate dehydrogenase in the TCA cycle and are essentially excluded from the glyoxylate cycle. Alternatively, the glyoxylate cycle contains two additional key enzymes: isocitrate lyase and malate synthase (Fig. 1). In brief, isocitrate lyase catalyses the breakdown of isocitrate $\left(C_{6}\right)$ into glyoxylate $\left(C_{2}\right)$ and succinate 
$\left(\mathrm{C}_{4}\right)$, followed by condensation of glyoxylate with acetyl$\mathrm{CoA}\left(\mathrm{C}_{2}\right)$ catalysed by malate synthase to generate malate $\left(\mathrm{C}_{4}\right)$ and a free $\mathrm{CoA}$ (Fig. 1). Therefore, malate produced from $\mathrm{C}_{2}$ compounds (e.g., ethanol and acetate) can serve as an intermediate to replenish the TCA cycle in the absence of glucose [71]. In addition, malate serves as the precursor of oxaloacetate, an essential substrate for gluconeogenesis. Oxaloacetate is converted to phosphoenolpyruvate by the gluconeogenesis enzyme phosphoenolpyruvate carboxykinase and eventually leads to glucose production with the help of another gluconeogenesis enzyme, fructose-1,6biphosphate (Fig. 1) [71]. In short, the glyoxylate cycle bypasses the two decarboxylation steps in the TCA cycle to allow for the anabolism of simpler carbon compounds in gluconeogenesis.

The glyoxylate cycle and its key metabolic enzymes, isocitrate lyase and malate synthase, are believed to be highly conserved across different organisms [67, 72]. The glyoxylate cycle plays an important role in the growth of plant seedlings and is involved in the conversion of stored lipids to carbohydrates, which serve as the main nutritional resource for the plant before the commencement of photosynthesis [73]. The glyoxylate cycle is also well studied in bacterial pathogens, especially in Mycobacterium tuberculosis. Honer $\mathrm{Zu}$ Bentrup et al. (1999) showed that M. tuberculosis possesses a second copy of the functional ICL1 gene (aceA) [53]. The glyoxylate cycle is reported to be essential for $M$. tuberculosis survival in the host because deletion of ICL1 and aceA genes leads to complete impairment of intracellular replication and rapid elimination of this pathogen from the lungs of C57BL/6 mice in vivo [74]. In addition, the glyoxylate cycle is essential for the pathogenicity of other intracellular bacterial pathogens, namely, Salmonella enterica serovar Typhimurium, Rhodococcus equi and Pseudomonas aeruginosa [75-77].

Inactivation of the ICL1 gene in the fungus Leptosphaeria maculans, a causal agent of the blackleg of crucifers, causes low germination rates of pycnidiospores, thus reducing the pathogenicity of this fungal pathogen on canola cotyledons [78]. In addition, the glyoxylate cycle is required for the full virulence of Magnaporthe grisea, the rice blast fungus. Disruption of ICL1 in $M$. grisea causes a reduction in appressorium formation, conidiogenesis and cuticle penetration, leading to an overall decrease in damage to rice and barley leaves [79]. Furthermore, disruption of MLS1 in the glyoxylate cycle also causes a reduction in the pathogenicity of certain plant pathogenic fungi, such as Rhodococcus fascians, Stagonospora nodorum and Xanthomonas campestris [80-82]. For intracellular human fungal pathogens, upregulation of glyoxylate cycle genes has been observed in Paracoccidioides brasiliensis, Penicillium marneffei, $C$. albicans, A. fumigatus and C. neoformans in response to macrophages [28, 83-86]. While the glyoxylate cycle is required for the full virulence of $C$. albicans, it is not essential for the full virulence of $A$. fumigatus and $C$. neoformans in vivo [87-89].

\section{Glyoxylate cycle and the virulence of C. glabrata: what we know so far}

The glyoxylate cycle enzymes isocitrate lyase and malate synthase are highly similar in C. glabrata and S. cerevisiae, with protein sequence identities of approximately 84 and $86 \%$, respectively. In contrast, both enzymes of C. glabrata and C. albicans have protein identities between 58 and 68\%, respectively. The subcellular localizations of isocitrate lyase and malate synthase have been inferred in the peroxisomes of C. albicans [90]. However, the isocitrate lyase of $S$. cerevisiae is localized in the cytosol, while malate synthase is compartmentalized in both the peroxisomes and the cytosol [70]. For C. glabrata, the subcellular localizations of both enzymes still remain to be determined, although WoLF PSORT predicts that these enzymes are localized in the peroxisomes, similar to $C$. albicans [91]. This result implies that $C$. glabrata could potentially regulate the localization of isocitrate lyase and malate synthase in a way that is similar to C. albicans, albeit with glyoxylate cycle enzymes that resemble those of baker's yeast.

The glyoxylate cycle in Candida species has been mainly studied in C. albicans, the predominant Candida species that causes invasive candidiasis in humans. Lorenz and Fink (2001) reported that the glyoxylate cycle genes ICL1 and MLS1 were significantly upregulated in C. albicans that were grown in the presence of macrophages [87]. In addition, the ICL1 gene is required for the full virulence of this fungal pathogen. Homozygous deletion of ICL1 rendered C. albicans avirulent in an in vivo mouse model of invasive candidiasis, while reintroducing ICL1 restored the virulence of this fungal pathogen in vivo. Genetic characterization of the glyoxylate cycle genes ICL1 and MLS1 revealed high homologies to other fungal species, particularly S. cerevisiae and C. tropicalis [92]. In addition, ICL1 and MLS1 were repressed in the presence of glucose and only induced in the presence of alternative carbon sources, which is very similar to the regulation of the glyoxylate cycle in S. cerevisiae.

Global gene expression analysis of $C$. albicans showed that ICL1 and MLS1 were also highly upregulated ( $>20$ fold) in response to human blood [93], which is in concordance with the data obtained from a previous study [87]. In 2004, transcriptional profiling of C. albicans in response to murine macrophages was investigated by using a microarray approach [28]. Microarray analyses revealed a reprogramming event in C. albicans in response to nutrient starvation in macrophages. In fact, genes related to alternative carbon metabolism, including genes from 
gluconeogenesis, glyoxylate cycles and $\beta$-oxidation of fatty acids, were found to be induced upon macrophage engulfment.

Barelle et al. (2006) revisited the role of the glyoxylate cycle in the virulence of $C$. albicans by using a green fluorescent protein (GFP) fusion approach instead of transcript profiling, which allows for the monitoring of individual C. albicans within host niches [94]. Indeed, ICL1-GFP fusion was repressed at physiologically relevant glucose concentrations $(0.1 \%)$ and only expressed in the presence of amino acids. Furthermore, induction of ICL1-GFP was observed in phagocytosed C. albicans in neutrophils and macrophages, but not in the nonphagocytosed cells. While most of the $C$. albicans cells expressed glycolytic genes PYK1 and PFK2 in the infected mouse kidneys, only half of the $C$. albicans cells expressed ICL1-GFP in the early stage of infection. This result implies that ICL1 is essential for the virulence of C. albicans in the early stage of infection in response to macrophages. However, a glycolytic mechanism is required for the disease progression of invasive candidiasis in the later stage of infection.

In addition, Ramírez and Lorenz (2007) reported that mutants lacking the glyoxylate cycle enzyme gene ICL1 displayed more extensive growth defects than initially predicted [95]. In addition to fatty acids, ethanol and acetate, ICL1 mutants of C. albicans are unable to grow in medium with citrate or glycerol as the sole carbon source. This finding suggests that the regulation of alternative carbon metabolism in C. albicans may be different compared to other fungi, such as $S$. cerevisiae. Ramírez and Lorenz (2007) also reconfirmed that ICL1 is required for the virulence of $C$. albicans by using a newly engineered strain that accounts for the positional effects of the URA3 disruption marker used in a previous study [87, 96]. Fernández-Arenas et al. (2007) used a proteomic approach to show that the glyoxylate cycle in C. albicans is upregulated in response to challenges from murine macrophages [97]. The glyoxylate cycle enzymes isocitrate lyase and malate synthase are localized to the peroxisome in a Pex5p-dependent manner. However, peroxisomal localizations of these enzymes are not essential for the functioning of the glyoxylate cycle, as this metabolic pathway works equally well in the cytosol of the cells [90].

Sandai et al. (2012) reported that there is significant disagreement between the proteome and transcriptome profiles of C. albicans [98]. Although the presence of glucose triggers the degradation of the ICL1 transcript, isocitrate lyase is stable and not destabilized, in contrast to the rapid destabilization of isocitrate lyase in S. cerevisiae following exposure to glucose. In fact, the isocitrate lyase of $C$. albicans lacks the main ubiquitination sites essential for targeted degradation of the transcript.
These findings suggest that $C$. albicans underwent posttranslational rewiring as it co-evolved with its host and thereby gained the ability to utilize alternative carbon sources and glucose simultaneously during systemic infection. Childers et al. (2016) investigated the impact of ubiquitin-mediated catabolite inactivation on the virulence of $C$. albicans [99]. The addition of ubiquitination sites in C. albicans leads to the destabilization of isocitrate lyase in response to glucose. Reduced metabolic flexibility following the addition of a ubiquitination site rendered $C$. albicans unable to use alternative carbon sources, more susceptible to macrophage killing, and unable to cause systemic and gastrointestinal tract infections in vivo.

As for C. glabrata, Kaur et al. (2007) have reported that the transcriptional response of C. glabrata coincubated with macrophages is very similar to the response described in C. albicans [29]. Genes involved in gluconeogenesis, the glyoxylate cycle, $\beta$-oxidation of fatty acids and the methylcitrate cycle were induced upon macrophage engulfment. In addition, Rai et al. (2012) revealed that alternative carbon remained the main intracellular carbon source in macrophages, and $C$. glabrata utilized acetyl-CoA via the glyoxylate cycle and gluconeogenesis for energy production [36]. Our previous work showed that the ICL1 gene is indeed crucial for the metabolism of alternative carbon sources in $C$. glabrata [100]. In fact, disruption of ICL1 rendered C. glabrata unable to utilize acetate, ethanol or oleic acid as the sole carbon source for growth and survival. In addition, ICL1 is also required for the intracellular survival of C. glabrata upon macrophage engulfment, potentially through the utilization of alternative carbon sources found within macrophages. Most importantly, ICL1 is essential for the virulence of C. glabrata in vivo, as disruption of ICL1 confers a severe attenuation in the virulence of $C$. glabrata in the mouse model of invasive candidiasis.

\section{Regulation of the glyoxylate cycle}

Most of the transcriptional regulators in yeast belong to a subclass of proteins known as zinc cluster proteins. These proteins are characterized by a well-conserved motif (CysX2CysX6CysX5-12CysX2CysX6-8Cys) and are fungal specific [101]. As most zinc cluster proteins bind to DNA, they are essential for the regulation of yeast transcriptional and translational machineries [102]. To date, transcriptional regulation of alternative carbon metabolism has not been well studied in C. glabrata. In S. cerevisiae, Snf1 kinase is the main activator of downstream glucose-repressed genes and is important for the activation of alternative carbon metabolism. Generally, Snf1 kinase is induced in response to glucose-deficient conditions and deactivates the transcriptional repressor 
Mig1 by phosphorylation [43]. Dissociation of Mig1 from the co-repressor protein complex Ssn6-Tup1 leads to increased expression of Cat8, a transcriptional regulator that binds the carbon source-responsive element (CSRE) located at the promoter region of many glyoxylate cycle and gluconeogenic genes such as ICL1, MLS1, FBP1 and PCK1 [43, 103, 104].

Cat8 is also an activator of Sip4, another important CSRE-binding transcriptional regulator found in S. cerevisiae. Although Cat8 and Sip4 have similar functions in regulating the glyoxylate cycle and gluconeogenic genes, Cat8 appears to be the primary regulator, because the removal of Cat8 significantly reduced the expression of CSRE-dependent genes, while the absence of Sip4 only caused a minor reduction in the expression of these genes $[105,106]$. Furthermore, activation of Sip4 requires a functional $C A T 8$, whereas activation of Cat8 is not dependent on SIP4. Unlike Mig1-regulated Cat8, activation of Sip4 is regulated by Snf1 Kinase and Rds2, another transcriptional regulator [43, 107]. Like Cat8 and Sip4, Rds2 also binds to the promoter region of CSRE-dependent genes from the glyoxylate cycle and gluconeogenesis following exposure to alternative carbon sources [107]. In short, CSRE-dependent genes from the glyoxylate cycle are at least recognized and regulated by three transcriptional regulators, namely, Cat8, Sip4 and Rds2, in S. cerevisiae.

Interestingly, removal of CAT8 did not alter the expression of CSRE-dependent genes that encode enzymes for the glyoxylate cycle, gluconeogenesis and $\beta$-oxidation of fatty acids in C. albicans [108]. Additionally, disruption of CAT8 did not lead to any noticeable attenuation in the virulence of $C$. albicans in vivo. This result suggests that Cat8 is not involved in the transcriptional regulation of glyoxylate cycle genes and is also not required for the full virulence of $C$. albicans. This observation could potentially be attributed to the substantial reassignment of the function of some transcriptional regulators between $C$. albicans and $S$. cerevisiae, which are reported to be highly divergent [109]. Therefore, it remains to be seen whether the regulation of the glyoxylate cycle in C. glabrata follows the more phylogenetically related $S$. cerevisiae or other Candida species from the CTG clade, such as C. albicans. Moreover, orthologous genes of SIP4 and RDS2 have been found in both C. albicans and C. glabrata [110, 111]; however, their involvement in the regulation of alternative carbon metabolism, particularly the glyoxylate cycle, remains unclear.

\section{Conclusion}

In conclusion, rapid and effective metabolic adaptation within the host is crucial for human fungal pathogens to thrive. The present review discussed the importance of alternative carbon metabolism as a metabolic adaptation strategy for intracellular C. glabrata to survive and propagate in carbon starvation. Considering the essential role of the glyoxylate cycle, particularly $I C L 1$, in the virulence of C. glabrata, further studies should be conducted to investigate other key metabolic enzymes involved in alternative carbon metabolism, notably those from gluconeogenesis and $\beta$-oxidation of fatty acids. Moreover, little is known about the transcriptional regulation and post-translation modification of these key metabolic enzymes in C. glabrata. Given the rapid emergence of invasive candidiasis caused by NCAC species, especially C. glabrata, it is envisaged that the knowledge generated from investigating this metabolic adaptation strategy will provide some basic insights into devising novel and innovative strategies for reducing the severity of invasive candidiasis caused by C. glabrata worldwide.

\section{Abbreviations}

BSIs: Bloodstream infections; CoA: Coenzyme A; CSRE: Carbon sources responsive element; DHAP: Dihydroxyacetone phosphate; GFP: Green fluorescent protein; ICU: Intensive care unit; NCAC: Non-C. albicans Candida; SCFAs: Short-chain fatty acids; SOT: Solid organ transplantation;

TCA: Tricarboxylic acid cycle.

\section{Acknowledgements}

Not applicable

Authors' contributions

SY, WC and LT reviewed the literature and co-wrote the manuscript. All authors listed gave final approval for publication.

\section{Funding}

This work was supported by Fundamental Research Grant Scheme (FRGS) from Ministry of Education (MOE), Malaysia (Grant number: 01-01-14-1456FR). S.Y. was supported by MyBrain 15 Scholarship from MOE, Malaysia. W.C. was supported by Graduate Research Fellowship (GRF) from Universiti Putra Malaysia.

Availability of data and materials

Not applicable

Ethics approval and consent to participate

Not applicable

Consent for publication

Not applicable

Competing interests

The authors declare that they have no competing interests.

Received: 17 April 2019 Accepted: 9 July 2019

Published online: 13 July 2019

\section{References}

1. Mayer FL, Wilson D, Hube B. Candida albicans pathogenicity mechanisms. Virulence. 2013;4:119-28.

2. Munoz-Duarte AR, Castrejon-Jimenez NS, Baltierra-Uribe SL, et al. Candida glabrata survives and replicates in human osteoblasts. Pathog Dis. 2016;74. ftw030.

3. Sadeghi G, Ebrahimi-Rad M, Mousavi SF, et al. Emergence of non-Candida albicans species: epidemiology, phylogeny and fluconazole susceptibility profile. J Mycol Med. 2018;28:51-8.

4. Pfaller MA, Diekema DJ. Epidemiology of invasive candidiasis: a persistent public health problem. Clin Microbiol Rev. 2007;20:133-63.

5. Andes DR, Safdar N, Baddley JW, et al. Impact of treatment strategy on outcomes in patients with candidemia and other forms of invasive 
candidiasis: a patient-level quantitative review of randomized trials. Clin Infect Dis. 2012:54:1110-22.

6. Falcone $\mathrm{M}$, Concia $\mathrm{E}$, lori l, et al. Identification and management of invasive mycoses in internal medicine: a road-map for physicians. Intern Emerg Med. 2014;9:501-11

7. Yapar N. Epidemiology and risk factors for invasive candidiasis. Ther Clin Risk Manag. 2014;13:95-105.

8. Taimur S. Yeast infections in solid organ transplantation. Infect Dis Clin N Am. 2018:32:651-66.

9. Kullberg BJ, Arendrup MC. Invasive candidiasis. N Engl J Med. 2015;373: 1445-56.

10. Fidel PL, Vazquez JA, Sobel JD. Candida glabrata: review of epidemiology, pathogenesis, and clinical disease with comparison to Candida albicans. Clin Microbiol Rev. 1999;12:80-96.

11. Messer SA, Jones RN, Fritsche TR. International surveillance of Candida spp. and Aspergillus spp.: report from the SENTRY antimicrobial surveillance program (2003). J Clin Microbiol. 2006;44:1782-7.

12. Messer SA, Moet GJ, Kirby JT, et al. Activity of contemporary antifungal agents, including the novel echinocandin anidulafungin, tested against Candida spp., Cryptococcus spp., and Aspergillus spp.: report from the SENTRY antimicrobial surveillance program (2006 to 2007). J Clin Microbiol. 2009:47:1942-6.

13. Pfaller MA, Diekema DJ, Gibbs DL, et al. Results from the ARTEMIS DISK global antifungal surveillance study, 1997 to 2007: a 10.5-year analysis of susceptibilities of Candida species to fluconazole and voriconazole as determined by CLSI standardized disk diffusion. J Clin Microbiol. 2010;48:1366-77.

14. Andes DR, Safdar N, Baddley JW, et al. The epidemiology and outcomes of invasive Candida infections among organ transplant recipients in the United States: results of the transplant-associated infection surveillance network (TRANSNET). Transpl Infect Dis. 2016;18:921-31.

15. Pfaller MA, Andes DR, Diekema DJ, et al. Epidemiology and outcomes of invasive candidiasis due to non-albicans species of Candida in 2,496 patients: data from the prospective antifungal therapy (PATH) registry 20042008. PLoS One. 2014;9:e101510.

16. Doi $A M$, Pignatari $A C C$, Edmond $M B$, et al. Epidemiology and microbiologic characterization of nosocomial candidemia from a Brazilian National Surveillance Program. PLoS One. 2016;11:e0146909.

17. Silva $S$, Negri M, Henriques M, et al. Candida glabrata, Candida parapsilosis and Candida tropicalis: biology, epidemiology, pathogenicity and antifungal resistance. FEMS Microbiol Rev. 2012;36:288-305.

18. Sardi JC, Scorzoni L, Bernardi T, et al. Candida species: current epidemiology, pathogenicity, biofilm formation, natural antifungal products and new therapeutic options. J Med Microbiol. 2013;62:10-24.

19. Wan L, Luo G, Lu H, et al. Changes in the hemolytic activity of Candida species by common electrolytes. BMC Microbiol. 2015;15:171.

20. Goyer $\mathrm{M}$, Loiselet $\mathrm{A}$, Bon $\mathrm{F}$, et al. Intestinal cell tight junctions limit invasion of Candida albicans through active penetration and endocytosis in the early stages of the interaction of the fungus with the intestinal barrier. PLoS One. 2016:11:e0149159.

21. Galocha M, Pais P, Cavalheiro M, et al. Divergent approaches to virulence in C albicans and C glabrata: two sides of the same coin. Int J Mol Sci. 2019;20: 2345.

22. Perlroth J, Choi B, Spellberg B. Nosocomial fungal infections: epidemiology, diagnosis, and treatment. Med Mycol. 2007;45:321-46.

23. Alves $C T$, Wei $X Q$, Silva $S$, et al. Candida albicans promotes invasion and colonisation of Candida glabrata in a reconstituted human vagina epithelium. J Inf Secur. 2014;69:396-407.

24. Tati S, Davidow P, McCall A, et al. Candida glabrata binding to Candida albicans hyphae enables its development in oropharyngeal candidiasis. PLoS Pathog. 2016;12:e1005522.

25. Duggan S, Essig F, Hünniger K, et al. Neutrophil activation by Candida glabrata but not Candida albicans promotes fungal uptake by monocytes. Cell Microbiol. 2015;17:1259-76

26. Seider K, Brunke S, Schild L, et al. The facultative intracellular pathogen Candida glabrata subverts macrophage cytokine production and phagolysosome maturation. J Immunol. 2011;187:3072-86.

27. Seider K, Gerwien F, Kasper L, et al. Immune evasion, stress resistance, and efficient nutrient acquisition are crucial for intracellular survival of Candida glabrata within macrophages. Eukaryot Cell. 2014;13:170-83.

28. Lorenz MC, Bender JA, Fink GR. Transcriptional response of Candida albicans upon internalization by macrophages. Eukaryot Cell. 2004;3:1076-87.
29. Kaur R, Ma B, Cormack BP. A family of glycosylphosphatidylinositol-linked aspartyl proteases is required for virulence of Candida glabrata. Proc Natl Acad Sci U S A. 2007;104:7628-33.

30. Roetzer A, Gratz N, Kovarik P, et al. Autophagy supports Candida glabrata survival during phagocytosis. Cell Microbiol. 2010;12:199-216.

31. Shimamura S, Miyazaki T, Tashiro M, et al. Autophagy-inducing factor Atg1 is required for virulence in the pathogenic fungus Candida glabrata. Front Microbiol. 2019;10:27.

32. Ene IV, Brunke S, Brown AJ, et al. Metabolism in fungal pathogenesis. Cold Spring Harb Perspect Med. 2014;4:a019695.

33. Brown AJ, Odds FC, Gow NA. Infection-related gene expression in Candida albicans. Curr Opin Microbiol. 2007;10:307-13.

34. Wilson D, Thewes S, Zakikhany $\mathrm{K}$, et al. Identifying infection-associated genes of Candida albicans in the postgenomic era. FEMS Yeast Res. 2009;9: 688-700.

35. Brown AJ, Brown GD, Netea MG, et al. Metabolism impacts upon Candida immunogenicity and pathogenicity at multiple levels. Trends Microbiol. 2014:22:614-22

36. Rai MN, Balusu S, Gorityala N, et al. Functional genomic analysis of Candida glabrata-macrophage interaction: role of chromatin remodelling in virulence. PLoS Pathog. 2012;8:e1002863.

37. Heymann P, Gerads M, Schaller M, et al. The siderophore iron transporter of Candida albicans (Sit1p/Arn1p) mediates uptake of ferrichrome-type siderophores and is required for epithelial invasion. Infect Immun. 2002;70: 5246-55.

38. Schrettl M, Haas H. Iron homeostasis - Achilles' heel of Aspergillus fumigatus? Curr Opin Microbiol. 2011;14:400-5.

39. Nevitt T, Thiele DJ. Host iron withholding demands siderophore utilization for Candida glabrata to survive macrophage killing. PLoS Pathog. 2011;7: e1001322

40. Sabina J, Brown V. Glucose sensing network in Candida albicans: a sweet spot for fungal morphogenesis. Eukaryot Cell. 2009:8:1314-20.

41. Ehrström S, Yu A, Rylander E. Glucose in vaginal secretions before and after oral glucose tolerance testing in women with and without recurrent vulvovaginal candidiasis. Obstet Gynecol. 2006;108:1432-7.

42. Miramón P, Lorenz MC. A feast for Candida: metabolic plasticity confers an edge for virulence. PLoS Pathog. 2017;13:e1006144.

43. Turcotte B, Liang XB, Robert F, et al. Transcriptional regulation of nonfermentable carbon utilization in budding yeast. FEMS Yeast Res. 2010;10:2-13.

44. Yamaguchi N, Sonoyama K, Kikuchi H, et al. Gastric colonization of Candida albicans differs in mice fed commercial and purified diets. J Nutr. 2005;135: 109-15.

45. Hohmann S. Osmotic stress signaling and osmoadaptation in yeasts. Microbiol Mol Biol Rev. 2002;66:300-72.

46. Beese-Sims SE, Pan SJ, Lee J, et al. Mutants in the Candida glabrata glycerol channels are sensitized to cell wall stress. Eukaryot Cell. 2012;11:1512-9.

47. Casal M, Paiva S, Andrade RP, et al. The lactate-proton symport of Saccharomyces cerevisiae is encoded by JEN1. J Bacteriol. 1999;181:2620-3.

48. Andrade RP, Kötter $\mathrm{P}$, Entian KD, et al. Multiple transcripts regulate glucose-triggered mRNA decay of the lactate transporter JEN1 from Saccharomyces cerevisiae. Biochem Biophys Res Commun. 2005;332: 254-62.

49. Lodi T, Ferrero I. Isolation of the DLD gene of Saccharomyces cerevisiae encoding the mitochondrial enzyme D-lactate ferricytochrome c oxidoreductase. Mol Gen Genet. 1993;238:315-24.

50. Lodi T, Diffels J, Goffeau A, et al. Evolution of the carboxylate Jen transporters in fungi. FEMS Yeast Res. 2007;7:646-56.

51. Ueno K, Matsumoto $Y$, Uno J, et al. Intestinal resident yeast Candida glabrata requires Cyb2p-mediated lactate assimilation to adapt in mouse intestine. PLoS One. 2011:6:e24759.

52. Strijbis K, Distel B. Intracellular acetyl unit transport in fungal carbon metabolism. Eukaryot Cell. 2010:9:1809-15.

53. Lorenz MC, Fink GR. Life and death in a macrophage: role of the glyoxylate cycle in virulence. Eukaryot Cell. 2002;1:657-62.

54. Paiva S, Devaux F, Barbosa $S$, et al. Ady $2 p$ is essential for the acetate permease activity in the yeast Saccharomyces cerevisiae. Yeast. 2004;21:201-10.

55. Casal M, Paiva S, Queirós O, et al. Transport of carboxylic acids in yeasts. FEMS Microbiol Rev, 2008:32:974-94.

56. Vieira N, Casal M, Johansson B, et al. Functional specialization and differential regulation of short-chain carboxylic acid transporters in the pathogen Candida albicans. Mol Microbiol. 2010;75:1337-54. 
57. Mota S, Alves R, Carneiro C, et al. Candida glabrata susceptibility to antifungals and phagocytosis is modulated by acetate. Front Microbiol. 2015;6:919.

58. Cunha DV, Salazar SB, Lopes MM, et al. Mechanistic insights underlying tolerance to acetic acid stress in vaginal Candida glabrata clinical isolates. Front Microbiol. 2017:8:259.

59. Fukuda Y, Tsai HF, Myers TG, et al. Transcriptional profiling of Candida glabrata during phagocytosis by neutrophils and in the infected mouse spleen. Infect Immun. 2013;81:1325-33.

60. Black PN, DiRusso CC. Yeast acyl-CoA synthetases at the crossroads of fatty acid metabolism and regulation. Biochim Biophys Acta. 1771;2007:286-98.

61. Rottensteiner $\mathrm{H}$, Theodoulou FL. The ins and outs of peroxisomes: coordination of membrane transport and peroxisomal metabolism. Biochim Biophys Acta. 1763;2006:1527-40.

62. Swiegers JH, Dippenaar N, Pretorius IS, Bauer FF. Carnitine-dependent metabolic activities in Saccharomyces cerevisiae: three carnitine acetyltransferases are essential in a carnitine-dependent strain. Yeast. 2001;18:585-95.

63. Prigneau O, Porta A, Maresca B. Candida albicans CTN gene family is induced during macrophage infection: homology, disruption and phenotypic analysis of CTN3 gene. Fungal Genet Biol. 2004;41:783-93.

64. Strijbis K, van Roermund CW, Visser WF, et al. Carnitine-dependent transport of acetyl coenzyme a in Candida albicans is essential for growth on nonfermentable carbon sources and contributes to biofilm formation. Eukaryot Cell. 2008;7:610-8.

65. Strijbis K, van Roermund CW, Hardy GP, et al. Identification and characterization of a complete carnitine biosynthesis pathway in Candida albicans. FASEB J. 2009;23:2349-59.

66. Zhou H, Lorenz MC. Carnitine acetyltransferases are required for growth on non-fermentable carbon sources but not for pathogenesis in Candida albicans. Microbiology. 2008;154:500-9.

67. Kondrashov FA, Koonin EV, Morgunov IG, et al. Evolution of glyoxylate cycle enzymes in Metazoa: evidence of multiple horizontal transfer events and pseudogene formation. Biol Direct. 2006;1:31.

68. Kunze M, Hartig A. Permeability of the peroxisomal membrane: lessons from the glyoxylate cycle. Front Physiol. 2013;4:204.

69. Kornberg HL. The role and control of the glyoxylate cycle in Escherichia coli. Biochem J. 1966;99:1-11.

70. Kunze M, Pracharoenwattana I, Smith SM, et al. A central role for the peroxisomal membrane in glyoxylate cycle function. Biochim Biophys Acta. 1763;2006:1441-52.

71. Dunn MF, Ramírez-Trujillo JA, Hernández-Lucas I. Major roles of isocitrate lyase and malate synthase in bacterial and fungal pathogenesis. Microbiol. 2009:55:3166-75.

72. Eastmond PJ, Germain V, Lange PR, et al. Postgerminative growth and lipid catabolism in oilseeds lacking the glyoxylate cycle. Proc Natl Acad Sci U S A 2000;97:5669-74.

73. Honer Zu Bentrup K, Miczak A, Swenson DL, et al. Characterization of activity and expression of isocitrate lyase in Mycobacterium avium and Mycobacterium tuberculosis. J Bacteriol. 1999;181:7161-7.

74. Muñoz-Elías EJ, McKinney JD. Mycobacterium tuberculosis isocitrate lyases 1 and 2 are jointly required for in vivo growth and virulence. Nat Med. 2005; 11:638-44.

75. Fang FC, Libby SJ, Castor ME, et al. Isocitrate lyase (AceA) is required for Salmonella persistence but not for acute lethal infection in mice. Infect Immun. 2005:73:2547-9.

76. Wall DM, Duffy PS, Dupont $C$, et al. Isocitrate lyase activity is required for virulence of the intracellular pathogen Rhodococcus equi. Infect Immun. 2005;73:6736-41

77. Lindsey TL, Hagins JM, Sokol PA, et al. Virulence determinants from a cystic fibrosis isolate of Pseudomonas aeruginosa include isocitrate lyase. Microbiol. 2008;154:1616-27

78. Idnurm A, Howlett BJ. Isocitrate lyase is essential for pathogenicity of the fungus Leptosphaeria maculans to canola (Brassica napus). Eukaryot Cell. 2002;1:719-24

79. Wang ZY, Thornton CR, Kershaw MJ, et al. The glyoxylate cycle is required for temporal regulation of virulence by the plant pathogenic fungus Magnaporthe grisea. Mol Microbiol. 2003;47:1601-12.

80. Vereecke D, Cornelis K, Temmerman W, et al. Chromosomal locus that affects pathogenicity of Rhodococcus fascians. J Bacteriol. 2002;184:1112-20.

81. Solomon PS, Lee RC, Wilson TJ, et al. Pathogenicity of Stagonospora nodorum requires malate synthase. Mol Microbiol. 2004;53:1065-73.
82. Tamir-Ariel D, Navon N, Burdman S. Identification of genes in Xanthomonas campestris pv. Vesicatoria induced during its interaction with tomato. J Bacteriol. 2007;189:6359-71.

83. Fan W, Kraus PR, Boily MJ, et al. Cryptococcus neoformans gene expression during murine macrophage infection. Eukaryot Cell. 2005;4:1420-33.

84. Ebel F, Schwienbacher M, Beyer J, et al. Analysis of the regulation, expression, and localization of the isocitrate lyase from Aspergillus fumigatus, a potential target for antifungal drug development. Fungal Genet Biol. 2006; 43:476-89.

85. Derengowski LS, Tavares AH, Silva S, et al. Upregulation of glyoxylate cycle genes upon Paracoccidioides brasiliensis internalization by murine macrophages and in vitro nutritional stress condition. Med Mycol. 2008;46: 125-34

86. Thirach S, Cooper CR Jr, Vanittanakom N. Molecular analysis of the Penicillium marneffei glyceraldehyde-3-phosphate dehydrogenase-encoding gene (gpdA) and differential expression of gpdA and the isocitrate lyaseencoding gene (acuD) upon internalization by murine macrophages. J Med Microbiol. 2008;57:1322-8.

87. Lorenz MC, Fink GR. The glyoxylate cycle is required for fungal virulence. Nature. 2001;412:83-6.

88. Rude $\mathrm{TH}$, Toffaletti DL, Cox GM, et al. Relationship of the glyoxylate pathway to the pathogenesis of Cryptococcus neoformans. Infect Immun. 2003;70: 5684-94.

89. Schöbel F, Ibrahim-Granet $O$, Avé $P$, et al. Aspergillus fumigatus does not require fatty acid metabolism via isocitrate lyase for development of invasive aspergillosis. Infect Immun. 2007;75:1237-44.

90. Piekarska K, Hardy G, Mol E, et al. The activity of the glyoxylate cycle in peroxisomes of Candida albicans depends on a functional beta-oxidation pathway: evidence for reduced metabolite transport across the peroxisomal membrane. Microbiol. 2008:54:3061-72.

91. Horton P, Park KJ, Obayashi T, et al. WoLF PSORT: protein localization predictor. Nucleic Acids Res. 2007:35:W585-7.

92. Eschrich D, Kötter P, Entian KD. Gluconeogenesis in Candida albicans. FEMS Yeast Res. 2002;2:315-25.

93. Fradin C, Kretschmar M, Nichterlein T, et al. Stage-specific gene expression of Candida albicans in human blood. Mol Microbiol. 2003:47:1523-43.

94. Barelle CJ, Priest CL, MacCallum DM, et al. Niche-specific regulation of central metabolic pathways in a fungal pathogen. Cell Microbiol. 2006;8: $961-71$

95. Ramírez MA, Lorenz MC. Mutations in alternative carbon utilization pathways in Candida albicans attenuate virulence and confer pleiotropic phenotypes. Eukaryot Cell. 2007;6:280-90.

96. Brand A, MacCallum DM, Brown AJ, et al. Ectopic expression of URA3 can influence the virulence phenotypes and proteome of Candida albicans but can be overcome by targeted reintegration of URA3 at the RPS10 locus. Eukaryot Cell. 2004;3:900-9.

97. Fernández-Arenas E, Cabezón V, Bermejo C, et al. Integrated proteomics and genomics strategies bring new insight into Candida albicans response upon macrophage interaction. Mol Cell Proteomics. 2007:6:460-78.

98. Sandai D, Yin Z, Selway L, et al. The evolutionary rewiring of ubiquitination targets has reprogrammed the regulation of carbon assimilation in the pathogenic yeast Candida albicans. mBio. 2012;3:e00495-12.

99. Childers DS, Raziunaite I, Mol Avelar G, et al. The rewiring of ubiquitination targets in a pathogenic yeast promotes metabolic flexibility, host colonization and virulence. PLoS Pathog. 2016;12:e1005566.

100. Chew SY, Ho KL, Cheah YK, et al. Glyoxylate cycle gene ICL 1 is essential for the metabolic flexibility and virulence of Candida glabrata. Sci Rep. 2019;9: 2843

101. MacPherson S, Larochelle M, Turcotte B. A fungal family of transcriptional regulators: the zinc cluster proteins. Microbiol Mol Biol Rev. 2006;70:583604.

102. Laity JH, Lee BM, Wright PE. Zinc finger proteins: new insights into structural and functional diversity. Curr Opin Struct Biol. 2001;11:39-46.

103. Schöler A, Schüller HJ. A carbon source-responsive promoter element necessary for activation of the isocitrate lyase gene $I C L 1$ is common to genes of the gluconeogenic pathway in the yeast Saccharomyces cerevisiae. Mol Cell Biol. 1994;14:3613-22.

104. Roth S, Kumme J, Schüller HJ. Transcriptional activators Cat8 and Sip4 discriminate between sequence variants of the carbon source-responsive promoter element in the yeast Saccharomyces cerevisiae. Curr Genet. 2004; $45: 121-8$ 
105. Vincent O, Carlson M. Sip4, a Snf1 kinase-dependent transcriptional activator, binds to the carbon source-responsive element of gluconeogenic genes. EMBO J. 1998;17:7002-8.

106. Hiesinger M, Roth S, Meissner E, et al. Contribution of Cat8 and Sip4 to the transcriptional activation of yeast gluconeogenic genes by carbon sourceresponsive elements. Curr Genet. 2001;39:68-76.

107. Soontorngun N, Larochelle M, Drouin S, et al. Regulation of gluconeogenesis in Saccharomyces cerevisiae is mediated by activator and repressor functions of Rds2. Mol Cell Biol. 2007;27:7895-905.

108. Ramírez MA, Lorenz MC. The transcription factor homolog CTF1 regulates beta-oxidation in Candida albicans. Eukaryot Cell. 2009;8:1604-14.

109. Martchenko M, Levitin A, Hogues $H$, et al. Transcriptional rewiring of fungal galactose-metabolism circuitry. Curr Biol. 2007:17:1007-13.

110. Maicas S, Moreno I, Nieto A, et al. In silico analysis for transcription factors with Zn (II)(2) C (6) binuclear cluster DNA-binding domains in Candida albicans. Comp Funct Genomics. 2005;6:345-56.

111. Klimova N, Yeung R, Kachurina N, et al. Phenotypic analysis of a family of transcriptional regulators, the zinc cluster proteins, in the human fungal pathogen Candida glabrata. G3. 2014;4:931-40.

\section{Publisher's Note}

Springer Nature remains neutral with regard to jurisdictional claims in published maps and institutional affiliations.

Ready to submit your research? Choose BMC and benefit from:

- fast, convenient online submission

- thorough peer review by experienced researchers in your field

- rapid publication on acceptance

- support for research data, including large and complex data types

- gold Open Access which fosters wider collaboration and increased citations

- maximum visibility for your research: over $100 \mathrm{M}$ website views per year

At BMC, research is always in progress.

Learn more biomedcentral.com/submissions 